\title{
Discrete breathers and Anderson modes: two faces of the same phenomenon?
}

\author{
J.F.R. Archilla ${ }^{\mathrm{a}, 1}$, R.S. MacKay ${ }^{\mathrm{b}, 2}$, J.L. Marín ${ }^{\mathrm{c}, 3}$ \\ ${ }^{a}$ Faculty of Computer Science, Avda Reina Mercedes, 41013-Sevilla, Spain \\ ${ }^{\mathrm{b}}$ The Nonlinear Centre, Department of Applied Mathematics and Theoretical \\ Physics, University of Cambridge, Silver Street, Cambridge CB3 9EW, U.K. \\ ${ }^{\mathrm{c}}$ Department of Mathematics, Heriot-Watt University, \\ Edinburgh EH14 4AS, U.K.
}

\begin{abstract}
Time-periodic localised oscillations occur in a variety of contexts, in particular in weakly coupled anharmonic lattices and in disordered harmonic networks of oscillators, where they are known respectively as discrete breathers and Anderson modes. It is shown numerically in some examples of systems which interpolate between these two limits that discrete breathers can be continued to Anderson modes, modulo small jumps associated with resonance with Anderson modes on other parts of the network.
\end{abstract}

Key words: Discrete breathers; Intrinsic localized modes; Anderson localization PACS: $63.20 . \mathrm{Pw}$

\section{Introduction}

Spatially localized time-periodic oscillations, or "localized oscillations" (LO) for short, appear in various types of spatially discrete models. In particular they occur in lattices of weakly coupled anharmonic oscillators and in disordered lattices of linear oscillators, but their origins in these two contexts have up till now been thought of as independent. For example, Scott's assessment of the numerics of Feddersen [1] on a disordered discrete self-trapping (DST)

1 Corresponding author. E-mail: archilla@cica.es

2 E-mail: r.s.mackay@damtp.cam.ac.uk

3 E-mail: jose@ma.hw.ac.uk 
equation is that "Anderson localization is completely different from anharmonic localization" (section 5.2 of [2]), because the numerics show Anderson modes delocalising rapidly as the anharmonicity is turned on. In our paper, however, it is shown numerically that on moving parameters in the opposite direction in a family of models, LOs of anharmonic ordered systems can be connected virtually continuously to LOs of harmonic disordered systems. First we recall the basic results in the two contexts.

LOs are common in anharmonic lattices and have received considerable attention recently (for a review, see [3]). They have been given a variety of names, but the one we shall use is "discrete breather", or more simply "breather". In particular, MacKay and Aubry [4] proved analytically the existence of breathers in Hamiltonian lattices of weakly coupled oscillators of nonlinear Klein-Gordon type - those with an anharmonic ${ }^{4}$ on-site potential. The breathers are proved to exist as smooth continuations of the trivial solutions at zero coupling where some oscillators are on periodic orbits and the remainder at rest. This limit has been called the anti-integrable or anti-continuous limit [7], but is most simply referred to as the "uncoupled limit". Proofs have also been given for some systems with no on-site potential, e.g. [8,9].

On the other hand, localization is also a physical phenomenon observed in the context of wave propagation through disordered media. By "disorder" for a classical lattice we mean that the local frequencies and/or couplings between oscillators are random. When the disorder is sufficiently large, certain types of waves become trapped and the spreading of any initial wave packet is anomalously slow or impossible. Instead, the eigenfunctions are spatially localized, so give localized modes that we call "Anderson modes", following the pioneering work of Anderson for Schrödinger equations with random potential [10-12].

In this paper we address the question raised in [13], whether there is a connection between these two different types of LO, i.e. if there is a continuous path that transforms a breather in an anharmonic ordered lattice into an Anderson mode in a harmonic disordered lattice. We study the question numerically, using a variant of the continuation method developed in [14,15].

Our models are of the form

$$
H=\sum_{n=1}^{m} \frac{1}{2} p_{n}^{2}+\frac{1}{2} \omega_{n}^{2} u_{n}^{2}-s u_{n}^{3}+\varepsilon \frac{1}{2} \sum_{n=1}^{m-1}\left(u_{n}-u_{n+1}\right)^{2}
$$

where $\varepsilon$ is fixed at 0.05 , the "site frequencies" $\omega_{n}$ are chosen randomly from a 2-point distribution of variable width $\rho$, and the "anharmonicity" $s$ is also

4 "Anharmonic" means that the period of oscillation varies with amplitude. A better term is "non-isochronous", cf. [5], because the harmonic oscillator is by no means the only potential for which the period is independent of amplitude, e.g. [6]. 
variable. We consider paths in the $(\rho, s)$ plane connecting the extremes $(1,0)$ (random harmonic) to $(0,1)$ (translation-invariant anharmonic). This type of model differs from that of [1] in three ways. Firstly, Feddersen's model has global phase rotation symmetry which gives it special features (e.g. conserved excitation "number" and existence of "stationary solutions" - discrete breathers with time-dependence purely of the form $\left.e^{\mathrm{i} \omega t}\right)^{5}$. Secondly, Feddersen has a disordered coupling matrix in addition to random site frequencies ${ }^{6}$. Thirdly, Feddersen's distribution of site frequencies is continuous rather than discrete. Further work will be required to assess the effects that such differences can make, but we note that Kopidakis and Aubry [17] have recently studied a similar model to ours but with a continuous distribution of site frequencies, and it will be interesting to compare their results.

There are some mathematical results on this problem. Continuation of Anderson modes to weak nonlinearity (equivalently, from infinitesimal to small amplitude in a nonlinear system) was studied mathematically by Albanese and Fröhlich [18]. They proved that Anderson modes have a "continuation" to a Cantor set of amplitudes whose complement has vanishing relative measure in the neighbourhood of zero amplitude. Their proof leaves gaps in the set of allowed amplitudes because of the possibility of resonance with nearby Anderson modes. Non-continuability across these gaps is a priori quite likely but we considered it interesting to investigate numerically to what extent there is a genuine obstacle.

In the other direction, the proofs of existence of discrete breathers apply equally well to disordered systems as to translation invariant lattices: simply, more care must be taken to satisfy the condition of non-resonance between the breather frequency and the linearised frequencies about the equilibria [19] (see also [13] for a special case). Thus continuation of discrete breathers from the ordered nonlinear regime is guaranteed to at least some amount of disorder and weakening of the nonlinearity.

The questions we address here numerically are whether one can find continuous paths of LOs from the ordered nonlinear regime to the edge of the AlbaneseFröhlich regime and whether there is a genuine obstacle to continuation within the Albanese-Fröhlich regime. The answer we find in our chosen model is that all the discrete breathers we studied continue to Anderson modes except for some small discontinuities occurring in the Albanese-Fröhlich regime.

\footnotetext{
5 DST models (also known as discrete nonlinear Schrödinger equations) were an important early source of LOs in nonlinear lattices [16], but the recent wave of activity on LOs is connected with the realisation that the phenomenon is much more general.

${ }^{6}$ He also kept the disorder fixed, but by scaling the field by the square root of the nonlinearity this is equivalent to decreasing the disorder to zero as the nonlinearity goes to infinity.
} 
Throughout the paper we use the term LO for Anderson modes, breathers and any time-periodic spatially localized oscillations in between. We avoid the terms "mode", which suggests a linear regime, and "intrinsic", which is supposed to denote an effect not requiring randomness, because our message is that localized oscillation is a more general phenomenon encompassing both limits.

We begin by describing the chosen model in Section 2. Then in Sections 3,4 and 5 , respectively, we describe the numerical methods used to obtain a breather in the anharmonic ordered case, to continue it towards the linear disordered case, and to compute its linear stability. The results are presented in Sections 6 and 7 . The paper concludes with a short summary in Section 8.

\section{The model}

We study an anharmonic Hamiltonian system of the Klein-Gordon type, given by the Hamiltonian:

$$
H=\sum_{n=1}^{m} \frac{1}{2} p_{n}^{2}+\frac{1}{2} \omega_{n}^{2} u_{n}^{2}-s u_{n}^{3}+\varepsilon \frac{1}{2} \sum_{n=1}^{m-1}\left(u_{n}-u_{n+1}\right)^{2}
$$

where $\left\{u_{n}\right\}_{n=1}^{m}$ are the coordinates of the $m$ particles with respect to their equilibrium positions; $\left\{\omega_{n}\right\}_{n=1}^{m}$ are the frequencies of small amplitude oscillation of the particles, which can be different as will be explained shortly; $-s u_{n}^{3}$ is the anharmonic part of the on-site potential for the particle $n$, therefore a softening potential; $s$ is a parameter which describes the degree of anharmonicity and takes its value in $[0,1], s=0$ being the harmonic case; and $\varepsilon$ is the coupling parameter, $\varepsilon=0$ corresponding to no coupling. The coupling potential is harmonic and nearest neighbour, though both these assumptions can be relaxed.

The disorder is implemented here by means of the curvatures $\omega_{n}^{2}$ of the local potentials at their minima. Since the masses have all been chosen equal to 1 , this gives rise to frequencies $\omega_{n}$. Suppose they take two values randomly distributed, say:

$$
\omega_{n}=\omega_{0}\left(1+\rho \frac{r_{n}}{2}\right) .
$$

Here $\rho$ is the disorder parameter, taking its value in $[0,1]$. At $\rho=0$, there is no disorder and all the frequencies are equal to $\omega_{0}$, which is taken to be 1 ; $\left\{r_{n}\right\}_{n=1}^{m}$ are the components of a random vector of \pm 1 . At $\rho=1$, the most disordered case, the frequencies are $0.5 \omega_{0}$ or $1.5 \omega_{0}$. The parameter $\rho$ is to be considered a monotonic function $\rho=\rho(s)$ of the nonlinearity $s$, with $\rho(0)=1$ 
and $\rho(1)=0$, giving a path from the anharmonic ordered case to the harmonic disordered case. Examples of these paths are

$$
\rho=1-s^{q}, \quad q>0 .
$$

We study paths with $q=1$ and $q=\frac{1}{4}$ in this paper (it might have been more natural to take only $C^{\infty}$ paths, but we were interested to investigate the effect of the $q=\frac{1}{4}$ approach to the disordered linear limit too, because of the small number of bifurcations on it).

We could have chosen random masses or random coupling strengths instead of or as well as random curvatures, or a continuous distribution for $\omega_{n}$ rather than a discrete one, but we think that our choice is a good first case to study. For a study of an example with continuous distribution of $\omega_{n}$, see [17]. Note also how this approach differs from that used in [1]: in that work, the degree of disorder was kept fixed while the nonlinearity was tunable; here we tune both. We suspect that this is more relevant than the possible differences arising from the two underlying models (a Hamiltonian lattice of classical oscillators vs. a discrete self-trapping equation). We consider only 1D chains, but it would also be interesting to study $2 \mathrm{D}$ or $3 \mathrm{D}$ models and models in which the randomness arises partially or completely from a random network of couplings.

The dynamical equations of the system are $\left(\dot{p}_{n}=\ddot{u}_{n}=-\partial H / \partial u_{n}\right)$ :

$$
F_{n}(u, s, \varepsilon)=\ddot{u}_{n}+\omega_{n}^{2} u_{n}-3 s u_{n}^{2}+\varepsilon\left(2 u_{n}-u_{n-1}-u_{n+1}\right)=0 .
$$

\section{Obtaining breathers and Anderson modes}

\subsection{Obtaining a breather}

Numerical methods for finding breathers are described in $[14,15,20]$. We work in the space of time-periodic, time-symmetric solutions of specified frequency $\omega_{\mathrm{b}}$, with continuous second derivative, which is denoted by $\mathcal{E}_{s}^{2}\left(\omega_{\mathrm{b}}\right)$. Therefore the functions $u_{n}(t)$ can be approximated by truncated Fourier series (rotating wave approximation) of the form:

$$
u_{n}(t)=\sum_{k=-k_{m}}^{k_{m}} z_{n}^{k} \mathrm{e}^{\mathrm{i} k \omega_{\mathrm{b}} t}=z_{0}+\sum_{k=1}^{k=k_{m}} 2 z_{n}^{k} \cos \left(k \omega_{\mathrm{b}} t\right) .
$$

The second equality holds because $z_{n}^{k}$ is real and $z_{n}^{k}=z_{-n}^{k}$, as a result of the operator in Eq. (5) being real and the time-symmetry of $u_{n}(t)$. 
Using the Newton-Raphson method, we first find a solution for the isolated oscillator with a given frequency $\omega_{\mathrm{b}}$, using as a seed the solution of the harmonic problem. The starting seed for obtaining a breather is a particle with this solution and the others at rest. By varying $\varepsilon$ from $\varepsilon=0$, with small steps, we are able to obtain a breather from the uncoupled limit.

\subsection{Obtaining Anderson modes}

This is a much simpler task. When the on-site potential is harmonic the dynamical equations of the system (Eq. (5)) can be written:

$$
\ddot{u}_{n}=-\omega_{n}^{2} u_{n}-\varepsilon\left(2 u_{n}-u_{n-1}-u_{n+1}\right),
$$

or in more compact notation:

$$
\ddot{u}=-\Omega u \text {. }
$$

Then it is simply a question of finding eigenvectors $u$ of the matrix $\Omega$ and checking whether they are spatially localized. In one dimension (cf. [21]), for arbitrarily weak disorder the spectrum of $\Omega$ consists of $m$ eigenvalues $\tilde{\omega}_{k}^{2}$ in $(0, \infty)$, whose eigenvectors $v^{k}$ are localized near $n=k$ and decay exponentially as $|n| \rightarrow \infty$. Thus equation (8) has periodic solutions:

$$
u_{n}^{k}(t)=\cos \left(\tilde{\omega}_{k} t\right) v_{n}^{k}, \quad \sin \left(\tilde{\omega}_{k} t\right) v_{n}^{k}
$$

As we are working in the space of time-reversible solutions, we are only concerned with the first set of solutions of Eq. (9). They are easy to obtain numerically. Two of them are shown in Fig. 1, which are the LOs at the end of the $q=1 / 4$ and $q=1$ paths.

\section{Continuation of localized oscillations}

Here we explain the method that we have used for continuing the breather in its path towards disorder. Sample results are presented in Sections 6 and 7.

The continuation cannot be done at constant frequency as there is no guarantee that at the harmonic limit there is any Anderson mode with the breather frequency (except for the trivial solution, $u_{n}=0, \forall n$ ). Instead, we choose to maintain constant the action of the loop,

$$
I=\sum_{n=1}^{m} \oint p_{n} d q_{n}=\sum_{n=1}^{m} \oint \dot{u}_{n} d u_{n}
$$



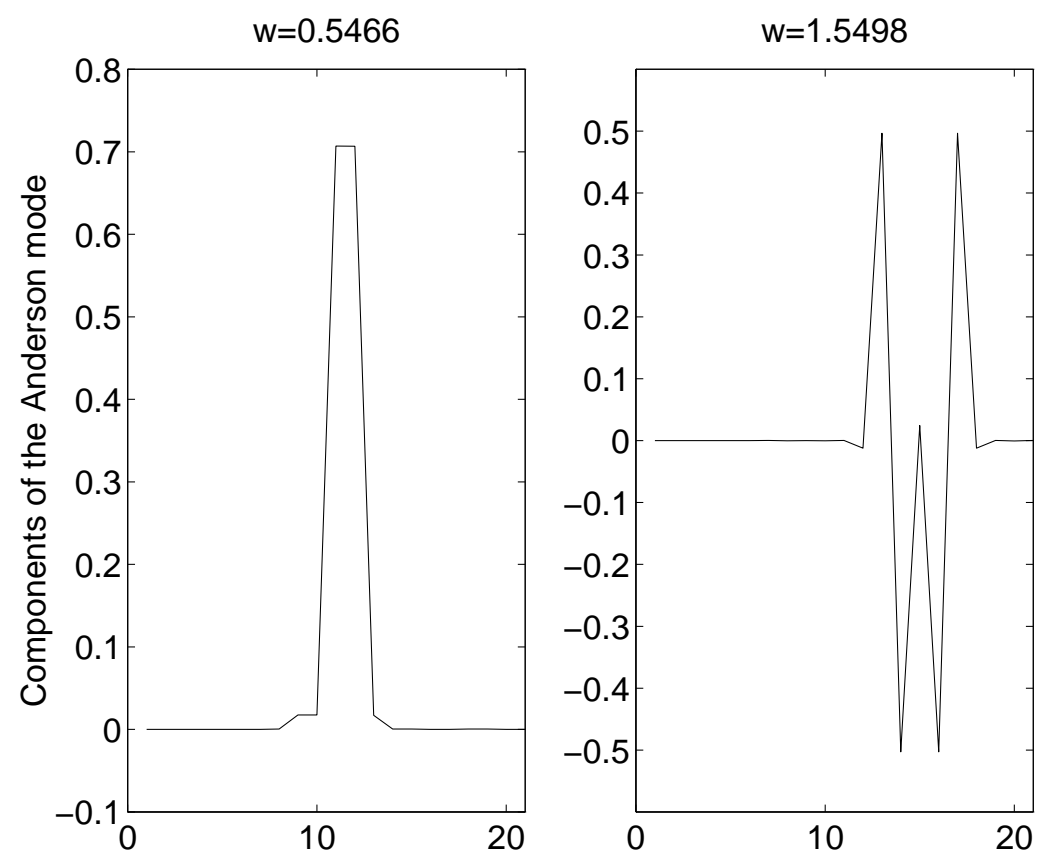

Fig. 1. The components of two Anderson modes, which are at the end of the paths with (a) $q=1 / 4$, and (b) $q=1$.

(perhaps better called its "area", as "action" has many other meanings). Among other advantages, this prevents the continuation from moving towards the zero solution. As the periodic functions of arbitrary frequency do not form a vector space, we change the time scaling: $t \rightarrow \phi=\omega_{\mathrm{b}} t$. Denoting differentiation with respect to $\phi$ by prime $\left(^{\prime}\right)$, we have $\ddot{u}=\omega_{\mathrm{b}}^{2} u^{\prime \prime}$, and $\omega_{\mathrm{b}}$ will appear in the dynamical function as a parameter to be determined. Therefore, we work in $\mathcal{E}_{s}(2 \pi) \times \mathbb{R}, \mathcal{E}_{s}(2 \pi)$ being a suitable Banach space of time-symmetric $2 \pi$-periodic functions. The action is $I=\omega_{\mathrm{b}} \sum_{n=1}^{m} \int_{0}^{2 \pi}\left(u_{n}^{\prime}\right)^{2} d \phi$.

Substituting the expressions (6) with $\phi=\omega_{\mathrm{b}}$ t into the dynamical equations (5), with $u^{\prime \prime}=\omega_{\mathrm{b}}^{2} \ddot{u}$, and collecting the terms in the exponentials $\exp (\mathrm{i} k \phi)$, we obtain $m\left(k_{m}+1\right)$ different algebraic equations $G(z, s)=\left\{\left\{G_{n}^{k}(z, s)\right\}_{k=0}^{k_{m}}\right\}_{n=1}^{m}$ for the $m\left(k_{m}+1\right)$ variables $z=\left\{\left\{z_{n}^{k}\right\}_{k=0}^{k_{m}}\right\}_{n=1}^{m}$ and the frequency of the selflocalized mode $\omega_{\mathrm{b}}$. Using the expressions for $u_{n}$ in Eq. (6), $I$ can be easily calculated to be

$$
I\left(z, \omega_{\mathrm{b}}\right)=\pi \omega_{\mathrm{b}} \sum_{n=1}^{m} \sum_{k=1}^{k_{m}}\left(k 2 z_{n}^{k}\right)^{2} .
$$

Then, we add to the system of equations $G$ the equation:

$$
G_{I}\left(z, \omega_{\mathrm{b}}\right)=I\left(z, \omega_{\mathrm{b}}\right)-I_{0}=0 .
$$

The dynamical equations are reduced to a set of $m\left(k_{m}+1\right)+1$ algebraic equations in the $m\left(k_{m}+1\right)+1$ unknowns $z_{n}^{k}$ and $\omega_{\mathrm{b}}$. Hereafter we will use 
the notation

$$
\tilde{G}=\left(G, G_{I}\right) \text { and } \tilde{z}=\left(z, \omega_{\mathrm{b}}\right) \text {. }
$$

The Jacobian of $\tilde{G}$ with respect of $\tilde{z}$ is of special importance. On the one hand its invertibility is the condition for the Implicit Function Theorem (IFT) to apply and, therefore, to guarantee the existence of a unique branch of solutions $\tilde{z}(s)$ in the neighbourhood of a solution $\tilde{z}_{0}$ at $s_{0}$. On the other hand, it is used within the Newton method for continuing a solution, which is the practical realisation of the IFT. If $\left(\tilde{z}_{0}, s_{0}\right)$ is a solution of $\tilde{G}(\tilde{z}, s)=0$, and $s_{1}=s_{0}+\delta s$ is a nearby value, writing $\tilde{z}_{1}=\tilde{z}_{0}+\delta \tilde{z}$, we have:

$$
\tilde{G}\left(\tilde{z}_{0}+\delta \tilde{z}, s_{1}\right) \approx \tilde{G}\left(\tilde{z}_{0}, s_{1}\right)+\partial_{\tilde{z}} \tilde{G}\left(\tilde{z}_{0}, s_{1}\right) \delta \tilde{z}
$$

Therefore, we obtain a first approximation for the solution at $s_{1}$ by making the last expression zero. This approximation is:

$$
\tilde{z}_{1}=\tilde{z}_{0}+\delta \tilde{z}=\tilde{z}_{0}-\left[\partial_{\tilde{z}} \tilde{G}\left(\tilde{z}_{0}, s_{1}\right)\right]^{-1} \tilde{G}\left(\tilde{z}_{0}, s_{1}\right) .
$$

Using $\tilde{z}_{1}$ as a new seed instead of $\tilde{z}_{0}$, and repeating this procedure, we are able to obtain a sequence of approximations to the solution at $s_{1}$ which, provided it exists and it is close enough to $s_{0}$, converges quadratically to machine precision.

We need an initial breather solution at $s_{0}=1$ to start our continuation procedure. This can be obtained by a preliminary continuation from the uncoupled limit, as discussed above in Section 3.1. In our numerics we started with the simplest configuration, single breathers (i.e. those which correspond in the uncoupled limit to one particle oscillating and the others at rest). Once the solution is continued to the desired value of the coupling $\varepsilon$, it becomes the starting point for our continuation into Anderson modes. We have used a model with 21 particles, a number which proves to be large enough to observe the LOs.

For every initial condition and path in parameter space that we examined, we found several values of $s$ where the Jacobian $\partial_{\tilde{z}} \tilde{G}$ has zero eigenvalues, so in principle a bifurcation could take place. In spite of that, the Newton method generated an almost continuous path of LOs.

To clarify whether an eigenvalue is really zero or only very small, because of the inaccuracy of the truncations of the Fourier series and the numerical error, we have plotted the whole set of eigenvalues with respect to $s$. Then it is easy 
to observe the evolution of the different eigenvalues, and if one of them changes sign it is clear that we have a zero eigenvalue. As the eigenvalues $\left\{v_{i}\right\}$ can be complex we plot $\operatorname{abs}\left(v_{i}\right) \operatorname{sign}\left(\operatorname{Re} v_{i}\right)$, which gives the clearest plot. A complex eigenvalue whose real part changes sign would appear as a discontinuity, but there are two of them, and they are easily recognized and checked with the plot of their counterpart abs $\left(v_{i}\right) \operatorname{sign}\left(\operatorname{Im} v_{i}\right)$.

Most of the eigenvalues of $\partial_{\tilde{z}} \tilde{G}$ are easily recognized, due to the fact that most oscillators in a LO are oscillating with very small amplitudes. An easy way to trace their origin is to analyze the spectrum from the limit of weak coupling. In such case, the dynamical equations corresponding to oscillators with small amplitudes are:

$$
F_{n}(u, s)=\omega_{\mathrm{b}}^{2} u_{n}^{\prime \prime}+\omega_{n}^{2}(s) u_{n}=0
$$

Substitution of the Fourier representation in terms of the modes $z_{n}^{k} \cos k \phi$, gives:

$$
-k^{2} \omega_{\mathrm{b}}^{2} z_{n}^{k}+\omega_{n}^{2}(s) z_{n}^{k}=0
$$

That is, these oscillators would only contribute with diagonal entries in the Jacobian $\partial_{\tilde{z}} \tilde{G}$, of the form:

$$
v_{n}^{k}=\omega_{n}^{2}(s)-k^{2} \omega_{\mathrm{b}}^{2}(s)
$$

As $\omega_{n}$ can take only two different values, $\omega_{ \pm}(s)=\omega_{0} \pm \frac{1}{2} \rho(s)$, we obtain:

$$
v_{ \pm}^{k}=\omega_{ \pm}^{2}(s)-k^{2} \omega_{\mathrm{b}}^{2}(s)
$$

Therefore, we obtain $2\left(k_{m}+1\right)$ different possible values for these eigenvalues. At $\varepsilon=0$, each of these has a degeneracy coinciding with the number of oscillators at rest with frequency $\omega_{+}(s)$ or $\omega_{-}(s)$, respectively. When the coupling $\varepsilon$ is switched on, each of these will split into a "band", and that degeneracy will generically be raised.

Then there will be other eigenvalues of different origin, those associated to sites which are in a high-amplitude oscillation (just one site for a single breather). Those will be typically detached from the bands discussed above, so they are easily spotted. Another complex conjugate pair of isolated eigenvalues comes from the last equation (Eq. 12). It is associated with the interchange of amplitude and frequency that maintains the action constant. 


\section{$5 \quad$ Linear Stability}

The linear stability of a LO can be calculated by means of the monodromy matrix, cf. $[22,7,20,19,15]$. This is obtained as follows; suppose $u=\left\{u_{n}(t)\right\}_{n=1}^{m}$ is an LO for a certain value of $s$, with frequency $\omega_{\mathrm{b}}$ and period $T_{b}=2 \pi / \omega_{\mathrm{b}}$ and $\xi(t)=\left\{\xi_{n}(t)\right\}_{n=1}^{m}$ a $\mathcal{C}_{m}^{2}$ small perturbation of $u(t)$ defined on $\left[0, T_{b}\right]$. Linearization of Eq. (5) gives the equations:

$$
\ddot{\xi}_{n}+\omega_{n}^{2} \xi_{n}-6 s u_{n}(t) \xi_{n}+\varepsilon\left(2 \xi_{n}-\xi_{n-1}-\xi_{n+1}\right)=0 .
$$

Writing the momenta $\pi_{n}(t)=\dot{\xi}_{n}(t)$, we obtain a set of dynamical equations for $\left(\left\{\xi_{n}(t)\right\},\left\{\pi_{n}(t)\right\}\right)$, which can be integrated numerically. As the functions $(\xi(t), \pi(t))$ are determined linearly by their initial conditions at $t=0$, and the integration determines their values at $t=T_{b}$, we obtain a $2 m \times 2 m$ linear operator, called the monodromy matrix, $T_{0}$ :

$$
\left(\begin{array}{c}
\left\{\xi_{n}\left(T_{b}\right)\right\} \\
\left\{\pi_{n}\left(T_{b}\right)\right\}
\end{array}\right)=T_{0}\left(\begin{array}{c}
\left\{\xi_{n}(0)\right\} \\
\left\{\pi_{n}(0)\right\}
\end{array}\right)
$$

Therefore the linear stability of the LO is determined by the eigenvalues of the monodromy matrix, called Floquet multipliers. The fact that the system is Hamiltonian and real implies that if $\lambda$ is an eigenvalue then $1 / \lambda, \lambda^{*}, 1 / \lambda^{*}$ are also eigenvalues. Then a necessary condition for linear stability is that all the Floquet multipliers be on the unit circle in the complex plane. Setting aside the cases with multiple Floquet multipliers of mixed symplectic signature, this is also a sufficient condition for linear stability.

\section{Results}

Here we give details and explanations of some numerical simulations, and present the results. The number of particles is $m=21$, the coupling parameter is $\varepsilon=0.05$. The number of Fourier coefficients was between 7 and 11, that is $k_{m} \in\{6, \ldots, 10\}$, which may appear small, but in fact we considered the truncation good only when the last Fourier coefficient is at least $10^{-6}$ times smaller than the largest one (which is always $k=1$ or 2 ). Of course, when we approach the disordered linear limit, this ratio diminishes to values of the order of $10^{-20}$. We have increased $k_{m}$ as a test to values up to 14 , but this does not produce any change (apart from the computational time), even at the difficult points where the eigenvalues of $\partial_{\tilde{z}} \tilde{G}$ approach zero and a change of behaviour is produced. The random vector of \pm 1 , which determines which 
particles have high or low frequency is:

$$
r_{n}=(1,-1,-1,-1,1,-1, \underbrace{1,1}_{A},-1,1, \underbrace{-1,-1}_{C}, \underbrace{1,1,-1,1,1}_{B},-1,-1,1,1),
$$

where we have labeled the groups of particles $A$ (particles 7 and 8), $C$ (particles 11 and 12), and $B$ (particles 13 to 17), for future reference. Several paths followed from nonlinearity-order, $s=1$, to linearity-disorder, $s=0$, have been studied. Also we have studied paths backwards starting at $s=0$, which usually lead to multi-breathers. Here we comment on two significant ones from $s=1$ : the linear one, where the function $\rho$ of Eq. (3) is $\rho=1-s$, and the path $\rho=1-s^{1 / 4}$, which we will refer to as the $1 / 4$ path.

\subsection{The linear path}

We start with the single breather with frequency $\omega_{\mathrm{b}}=0.85$ obtained from the uncoupled limit coded 0 for all the particles, except the central particle, number 11 , coded +1 . The code means 0 for particles at rest, +1 , for particles oscillating with phase 0 at $t=0$, and -1 , for initial phase $\pi$ (see for example [7]). This frequency allows a relatively large window of values outside the linear modes (the same reference). The Fourier spectrum of this breather appears in Fig. 2, and its projection onto the local phase planes in Fig. 3. Its action (area) was found to be $I=0.1286$.

Then we continued it from $s=1$ towards $s=0$. As $s$ diminishes, the two particles $\mathrm{C}$ with the same frequency at rest begin to oscillate in phase, evolving to what we will call the double central LO, that is the two particles $\mathrm{C}$ oscillating in phase and the others almost at rest. At the same time two new LOs appear; one of them consists of the group $\mathrm{B}$, oscillating with code $(+1,-1,0,-1,+1)$, and the other is the group A of particles with code $(-1,+1)$. All these oscillations can be seen in the Fourier spectrum that appears in Fig. 4. At $s_{1}=0.322$, the oscillation $\mathrm{C}$ disappears. At $s_{2}=0.234$ the oscillation $\mathrm{A}$ is annihilated and, in the end, the only surviving LO is B.

In Fig. 5 we can see the evolution of the eigenvalues of $\partial_{\tilde{z}} \tilde{G}(\tilde{z}, s)$. At smaller scale it can be seen that only the group of eigenvalues labelled $w 1-$ crosses the zero line, which happens about $s_{1}$. Inspection of the Fourier components shows that for $s<s_{1}$, the odd Fourier components are negligible, therefore the frequency is in fact doubled: we have an inverse period doubling bifurcation as $s$ decreases, and the resulting LO for $s<s_{1}$ is an orbit of half the action taken twice over.

The eigenvalues of the monodromy matrix evolve along the path, as shown in 


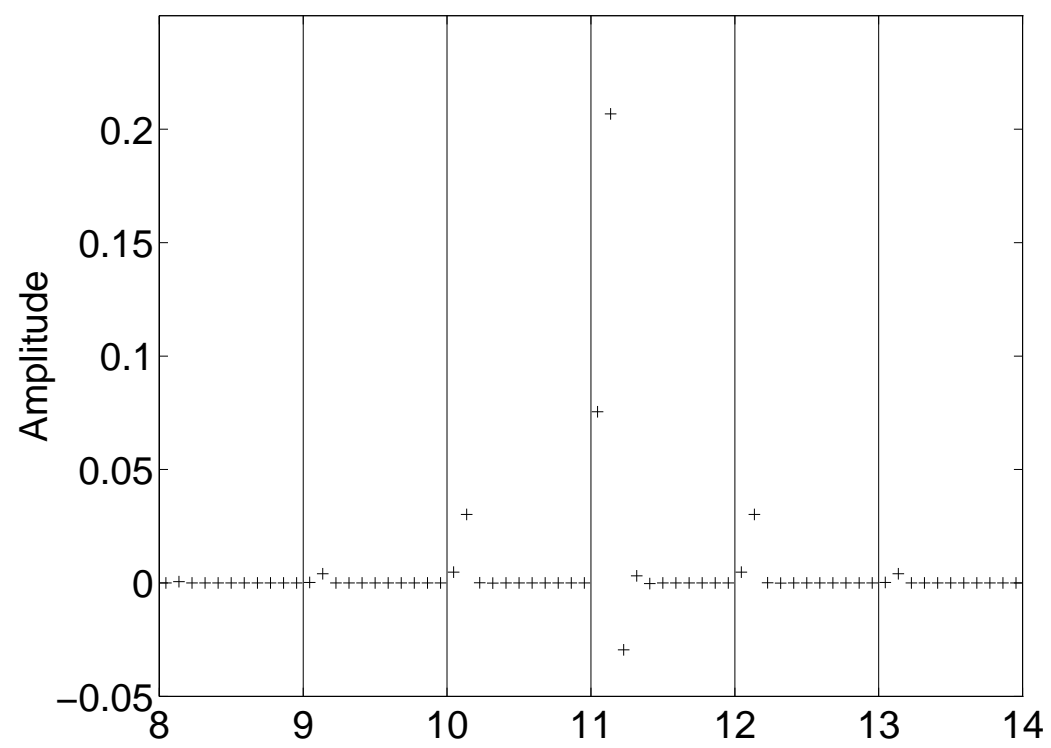

Fig. 2. Fourier components of the breather with $\omega_{b}=0.85$ and $s=1$. Vertical lines separate the coefficients of the particles. Crosses give the Fourier coefficients. We can see that only the first two or three of each particle (four for the eleventh one) contribute significantly to the spectrum.

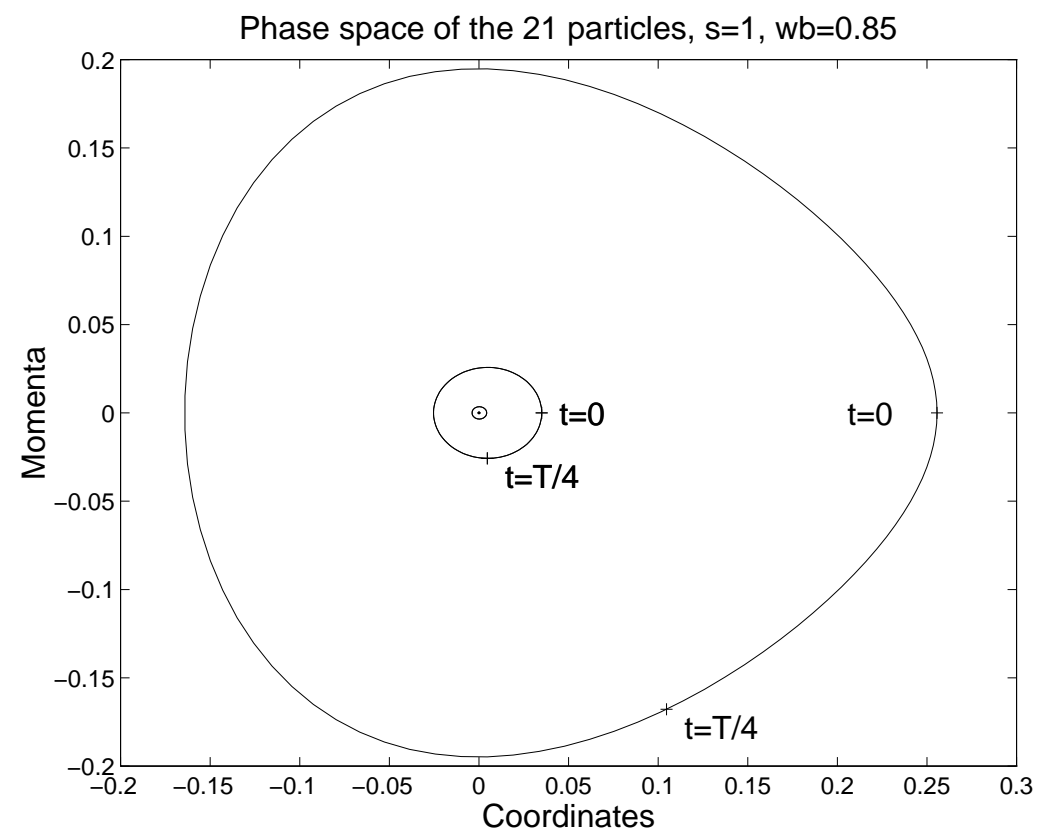

Fig. 3. Phase space of the breather. The exterior curve corresponds to the particle 11 , the first interior curve corresponds to both the two neighbouring particles.

Fig. 6. To numerical resolution they sometimes cross each other but do not appear to abandon the unit circle, even when some with opposite symplectic (Krein) signature [7] cross, or when a pair of conjugates join at \pm 1 . If observed on a small scale, however, we expect that one would see small bubbles of 


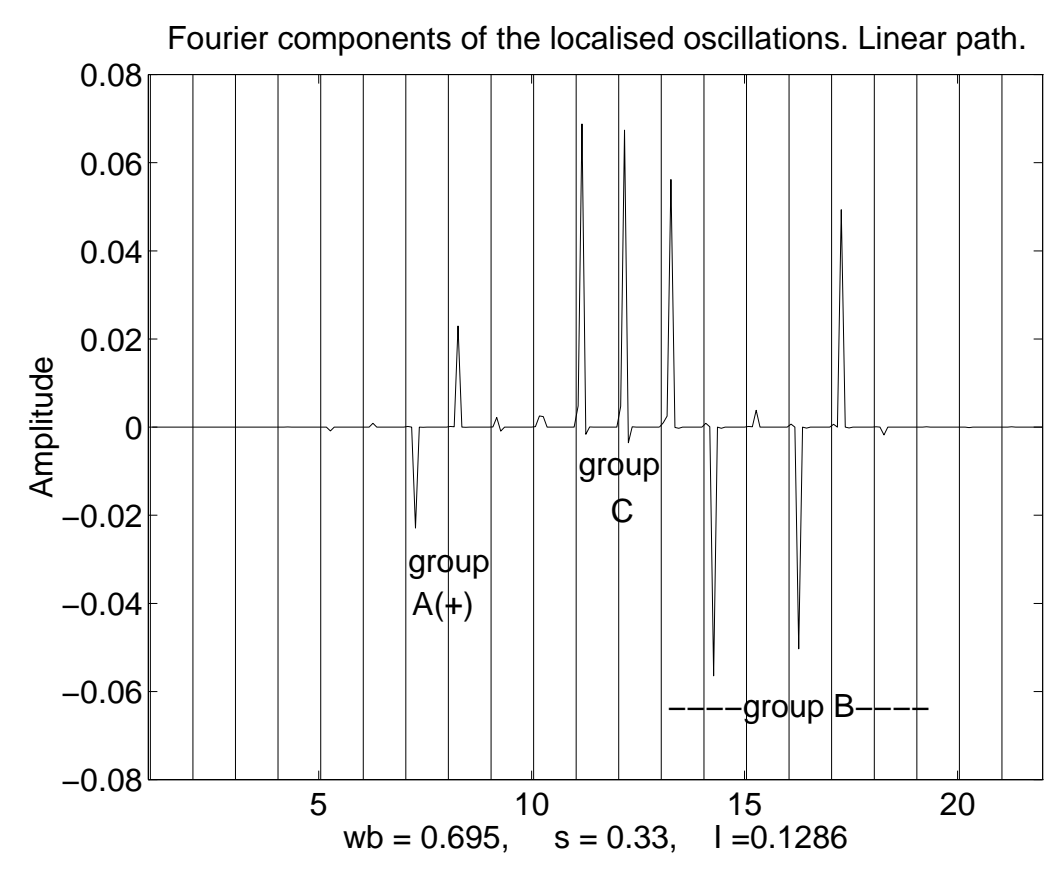

Fig. 4. LO at halfway to the disordered linear regime. From the single breather a two particle LO, C, is formed at 11 and 12, which will completely disappear at $s=0.322$, bif. 1 . The two particle LO, C, at particles 7 and 8 , will disappear at $s=0.234$, bif. 2 . There is a 5 particle LO, B, at $(13,14,15,16,17)$ which in the end will be completely symmetrized and the only surviving localized oscillation of non negligible amplitude. For these last two modes the largest Fourier component is $k=2$, and the odd $k$ 's are negligible. Therefore their frequency is in fact double that shown - we are close to a period doubling bifurcation.

instability near these collisions, cf. [15,20,23].

\subsection{The $1 / 4$ path}

This path starts with the same single breather, at the particle 11, but the evolution of the LO is much simpler. The neighbouring particle 12 begins to oscillate in phase, and we get a double LO in $\mathrm{C}$ that evolves to the double Anderson mode in $\mathrm{C}$. The evolution of the eigenvalues of the Jacobian can be observed in Fig. 7, with an enlargement in Fig. 8. The eigenvalue crossing zero at $s_{c}=0.2938$ can be identified with resonance with a linearised mode on particles 1-6; the oscillation of this group increases as $s$ approaches the $s_{c}$ from either side, but with opposite phase on the two sides, as illustrated in Fig. 9. Also, the whole group labelled $w 2+$ crosses zero near $s=0.01$, as mentioned in the figure caption. 


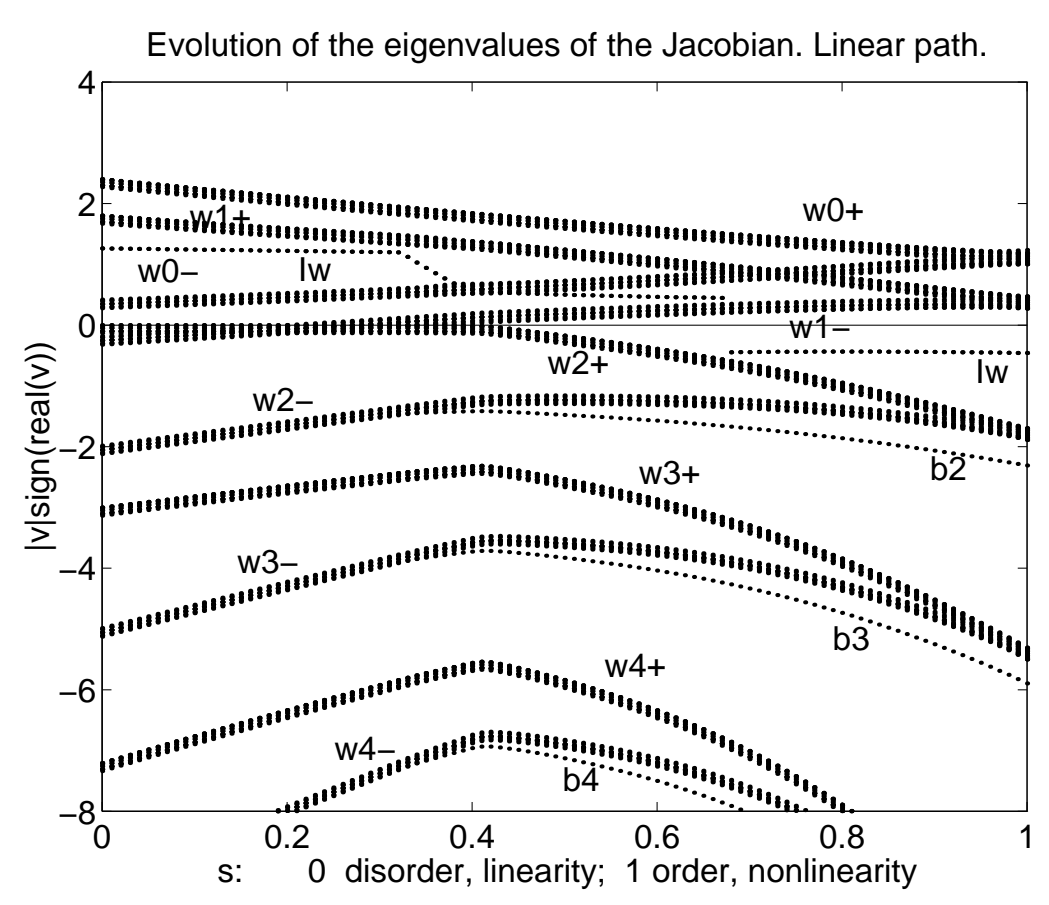

Fig. 5. Evolution of the eigenvalues of the Jacobian $\partial_{\tilde{z}} \tilde{G}(\tilde{z}, s)$. Most of the eigenvalues correspond to linear modes on the particles close to rest; these are labelled by $w k \pm$, where $k \in \mathbb{Z}$ denotes the dominant temporal Fourier harmonic involved and the \pm sign indicates whether the mode is associated with particles with disorder variable $r_{n}= \pm 1$, see eq. (2). The eigenvalues associated with perturbation of the excited sites are labeled $b k$; however, the first two are mixed with the linear modes. Finally, the eigenvalue labelled $I w$ is a complex conjugate pair related to the interchange of amplitude and frequency that maintains the action constant; the apparent discontinuity is due to the change of sign of the real part.

\section{Bifurcation study}

We have studied in some detail the important bifurcations, that is those that involve changes of behaviour of non-negligible amplitude. These bifurcations can be detected in several ways: slowness and/or difficulty of convergence of the Newton method, including jumps in the step prescribed; looking at the points where an eigenvalue of the Jacobian crosses or approaches zero; and abrupt changes in the Fourier spectrum or in the plot of the coordinates versus time. Special care has to be taken when an eigenvalue approaches zero and turns back. Is the Newton method jumping to another branch of solutions, or are we dealing with a bifurcation? With our present program, we cannot expect to reach the exact point where a bifurcation takes place, as the Jacobian is not invertible there (this could be solved by using arc-length continuation with a suitable choice of transversal). A great help is obtained, however, by restarting the Newton method after the bifurcation, and tracing it backwards, seeing if the solutions take the same path or another one, and in this case, which one. 


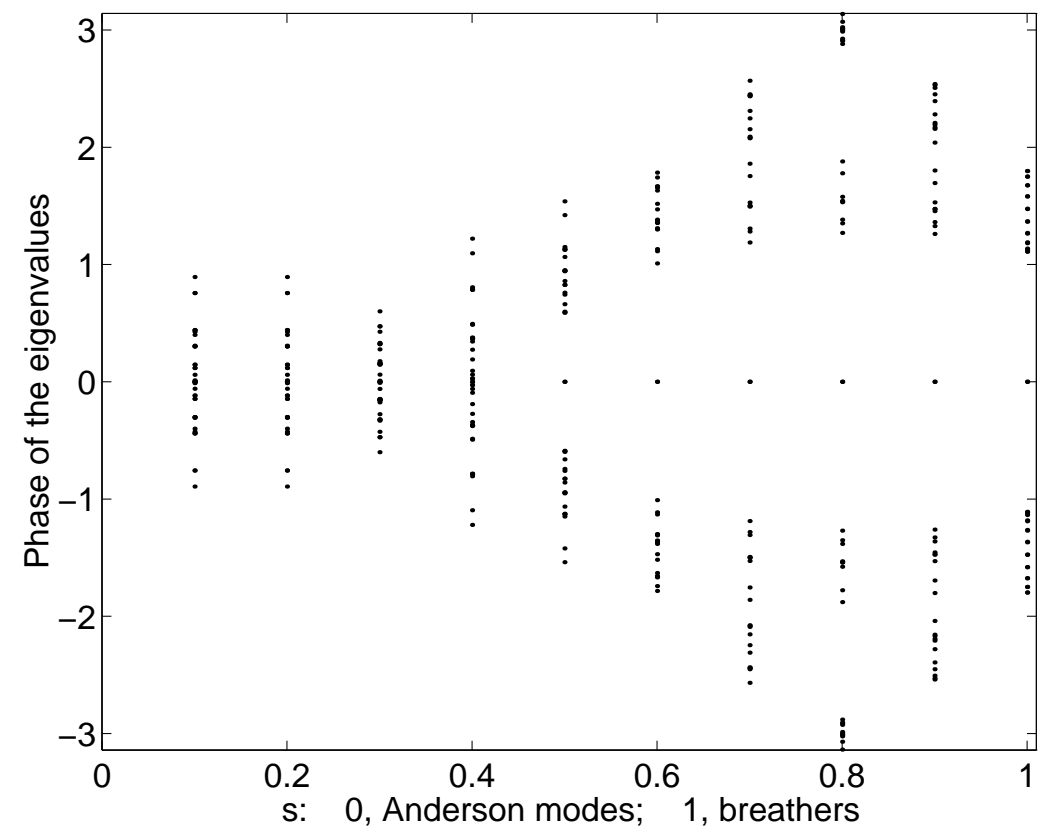

Fig. 6. Evolution of the eigenvalues of the monodromy for the linear path. All of them appear to remain of modulus 1 . The two groups of eigenvalues at $s=1$ which have different Krein signature cross around $s=0.8$ at -1 (phase $\pi$ ) and again mix about $s=0.4$ at 1 (phase 0 ) without apparent loss of stability.

Some other considerations such as approximate symmetries can be used, as we shall see below.

We depict bifurcation diagrams by plotting with respect to the parameter $s$ a bifurcation variable, which is the projection of the solutions on the null subspace of the Jacobian at the bifurcation, that is the centre subspace. If we do not have exactly zero eigenvalues, or two very small ones crossing or approaching zero, we may have to decide which is the appropriate centre subspace where the bifurcation is taking place. This can be done by comparing the corresponding eigenvectors just before and after the bifurcation and observing the evolution of the eigenvalues.

\subsection{Bifurcations in the $1 / 4$ path}

This is a very simple path as there is only an isolated eigenvalue crossing zero (Fig. 8), except when we get very close to the linear disordered case $(s=0.01)$, where a group of linear modes crosses zero. We think that the bifurcation associated with this eigenvalue crossing zero is a typical feature for LOs in random systems, when they resonate with linear modes on other parts of the system. The bifurcation diagram can be seen in Fig. 10. To understand this, 


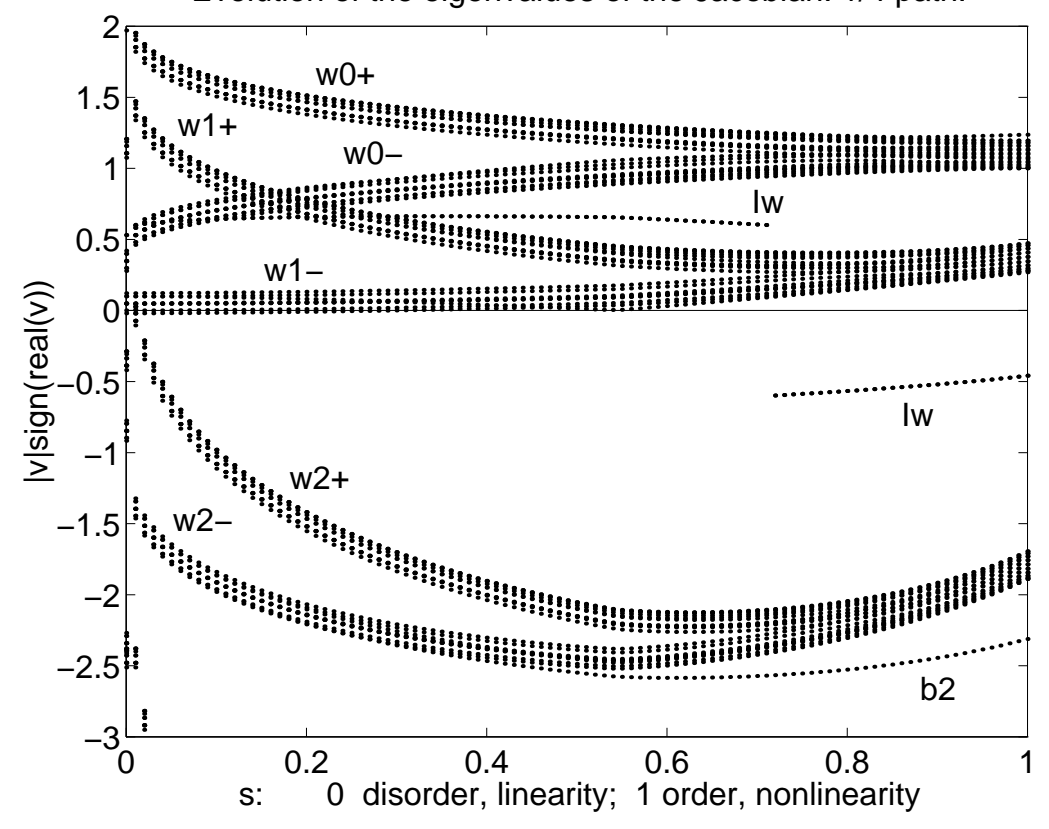

Fig. 7. Evolution of the eigenvalues of the $\partial_{\tilde{z}} \tilde{G}(\tilde{z}, s)$ along the $1 / 4$ path. The labels are the same as in Fig. 5 .

it is helpful to compare with Fig. 9 and to notice how the frequencies of the LO and a linearised mode located mainly on particles 2-4 cross (Fig. 11).

If the frequencies of a (time-symmetric) LO of action $I$ on one finite chain of oscillators and a linearised mode on another finite chain cross as a parameter $s$ passes through a value $s_{c}$, then for the joint system of two independent chains there is a pitchfork bifurcation in the set of time-symmetric LOs of action $I$ at $s=s_{c}$, because one can add any small amount of the linearised mode, in either phase, and decrease the action of the LO correspondingly. Generically, these changes cause a slight shift in the frequency ratio, requiring a compensating shift in parameter leading to a pitchfork. If one now connects the two chains by coupling their ends to make a single chain, the pitchfork can be expected generically to break (unless some spatial symmetry is preserved), making an imperfect pitchfork. This can be seen in the bifurcation diagram in Fig. 10. We have obtained the two outer prongs of the pitchfork by the following procedure: we add to a solution $\left\{u_{n}\right\}$, not too close to the bifurcation, the eigenvector corresponding to the centre subspace, scaled so as both have the same action. We rescale again so as to obtain a vector with the same action as our solutions. This is used as a seed for the Newton method to obtain a branch of solutions that joins to the previous one at the bifurcation point. Subtracting the eigenvector, instead of adding, we are able to obtain the other branch.

This can be confirmed easily by comparing the plots of the coordinates versus 


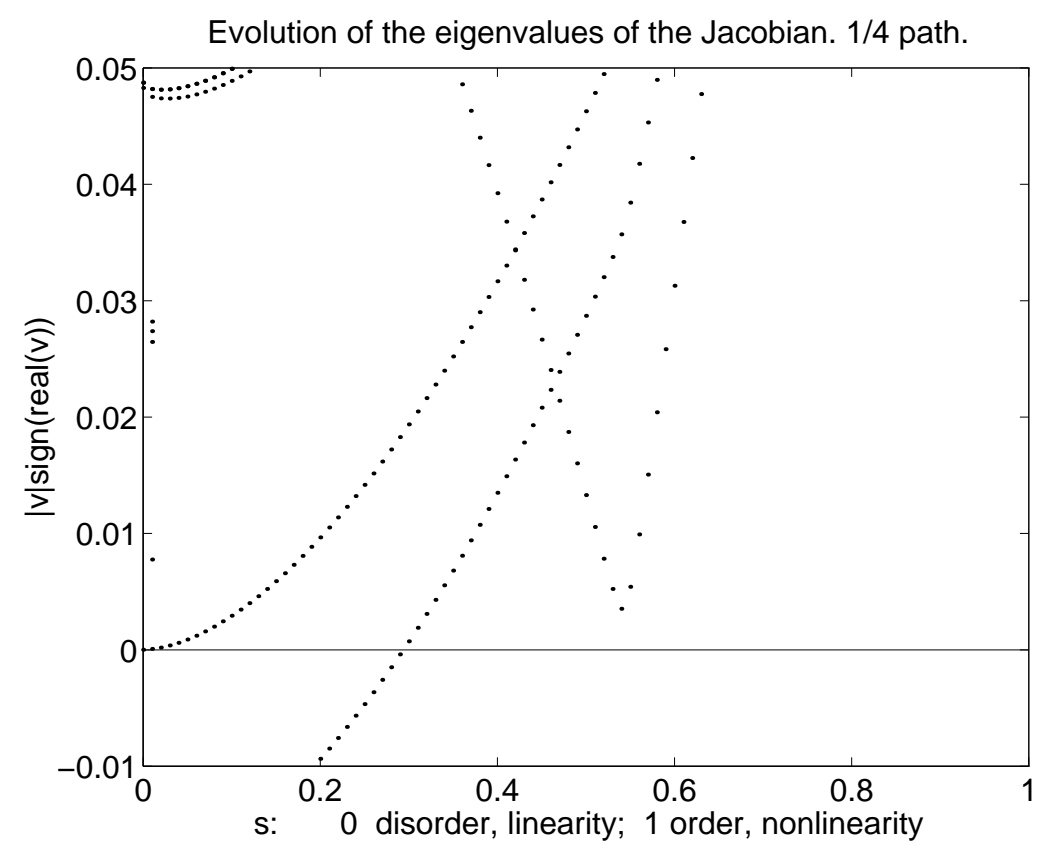

Fig. 8. Enlargement of Fig. 7, showing the eigenvalues in the group $w 1-$. Apparently only one eigenvalue crosses the zero line; it is related to a change of phase of a small amplitude mode on particle 5. The double LO on particles 11 and 12 evolves continuously. Also visible in this picture is the group of eigenvalues $w 2+$ at very small $s$, which crosses the zero line at about $s=0.01$; this is related to the disappearance of the Fourier components with $k \neq 1$ at the linear limit.

time before and after the bifurcation. It shows the change of phase in a group of six particles. Moreover, the null eigenvector is composed mainly of the first Fourier components of these particles.

This is not a standard imperfect pitchfork bifurcation, however, as both the "horizontal" branches are observed to be linearly stable, in contrast to a standard imperfect pitchfork for periodic orbits of a Hamiltonian system for which the "horizontal" branch would change stability. We conjecture that the bifurcation diagram should be completed by creation of a pair of unstable timeasymmetric LOs by a "Rimmer bifurcation" (pitchfork for periodic orbits of reversible Hamiltonian systems) [24] from one of the "vertical branches" after a short interval of instability.

Note that although the standard setting for analyzing bifurcations of periodic orbits in Hamiltonian systems is to consider variation of the set of periodic orbits of given energy $E$ with respect to $E$ and/or external parameters, the same results apply for given action $I$, because $\mathrm{d} I / \mathrm{d} E=T$, the period, which is neither zero nor infinite. 

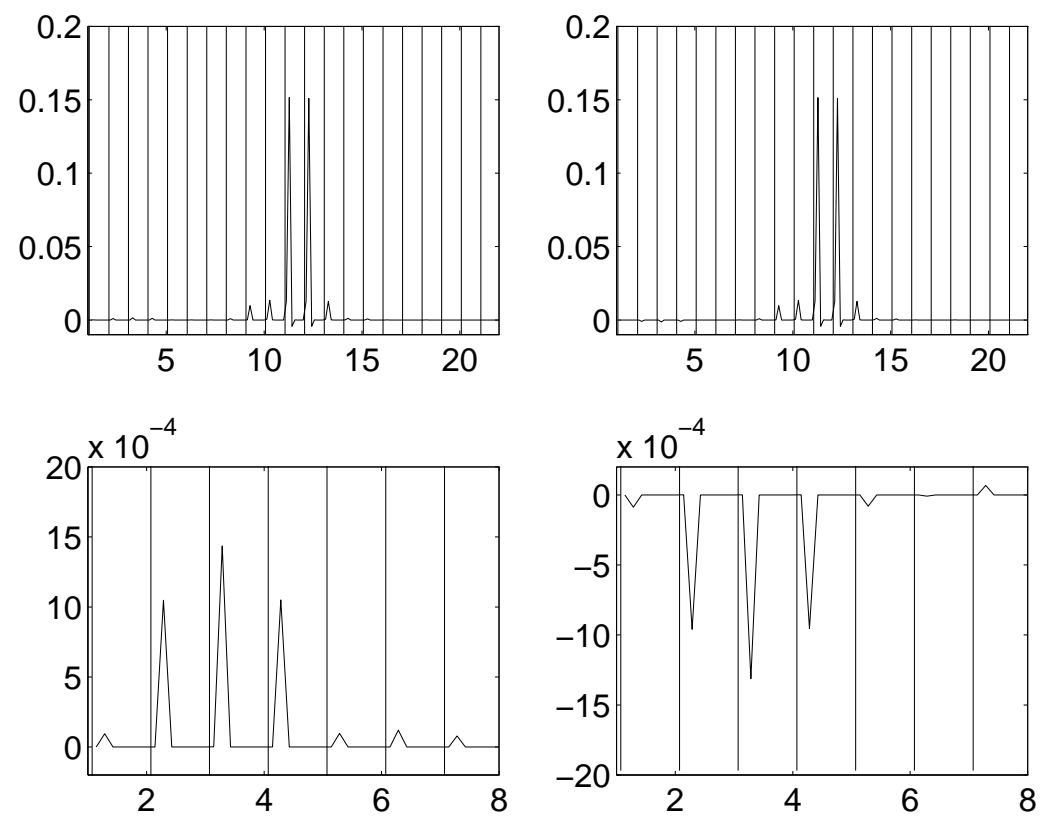

Fig. 9. Evolution of the LO through the zero eigenvalue at $s=0.2938$ on the $1 / 4$ path. On the right, Fourier components before the bifurcation, at $s=0.294$, on the left, after the bifurcation, at $s=0.293$. On the top, the 21 particles, on the bottom, particles 1-6.

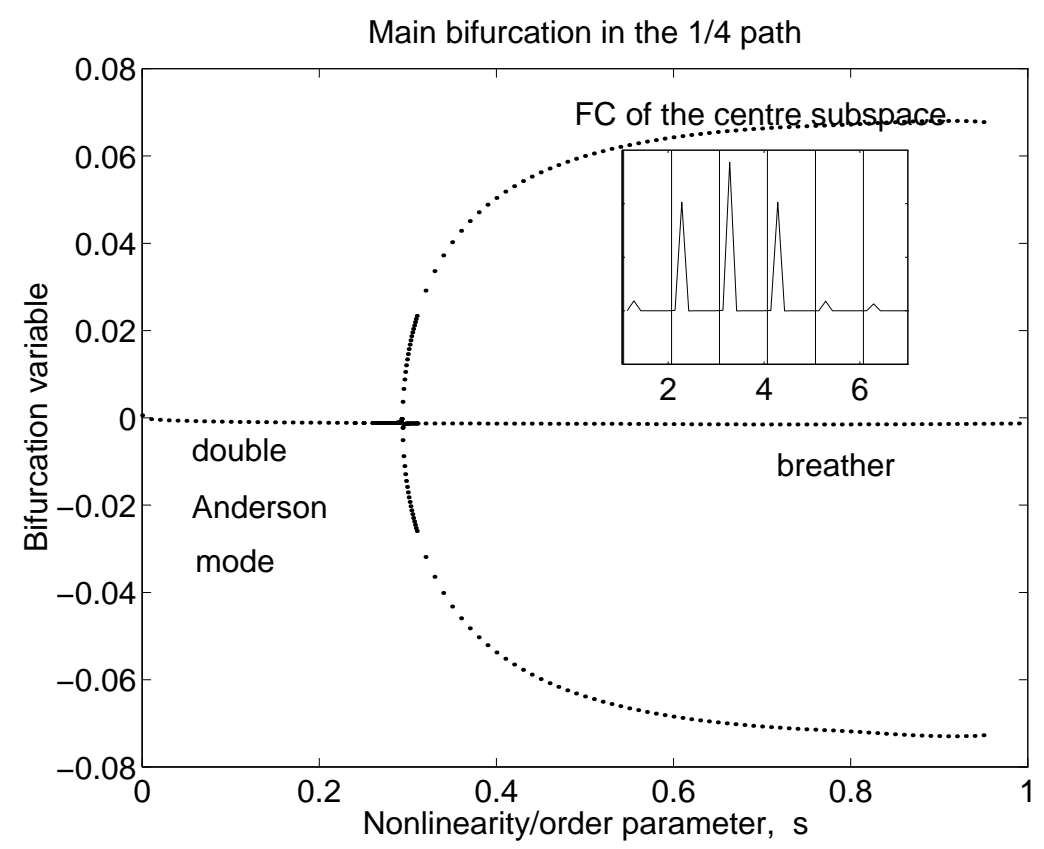

Fig. 10. Bifurcation diagram along the $1 / 4$ path. The bifurcation variable is the component in Fourier space in the direction of the eigenvector with zero eigenvalue for the operator $\partial_{\tilde{z}} \tilde{G}(\tilde{z}, s)$ at $s=0.2938$, which is shown in the inset. The outer prongs, composed of the central oscillation plus an oscillation similar to this eigenvector, with the same phase for the upper prong, and a phase difference of $\pi$ for the lower one, lead to non localized oscillations in the nonlinear limit. 


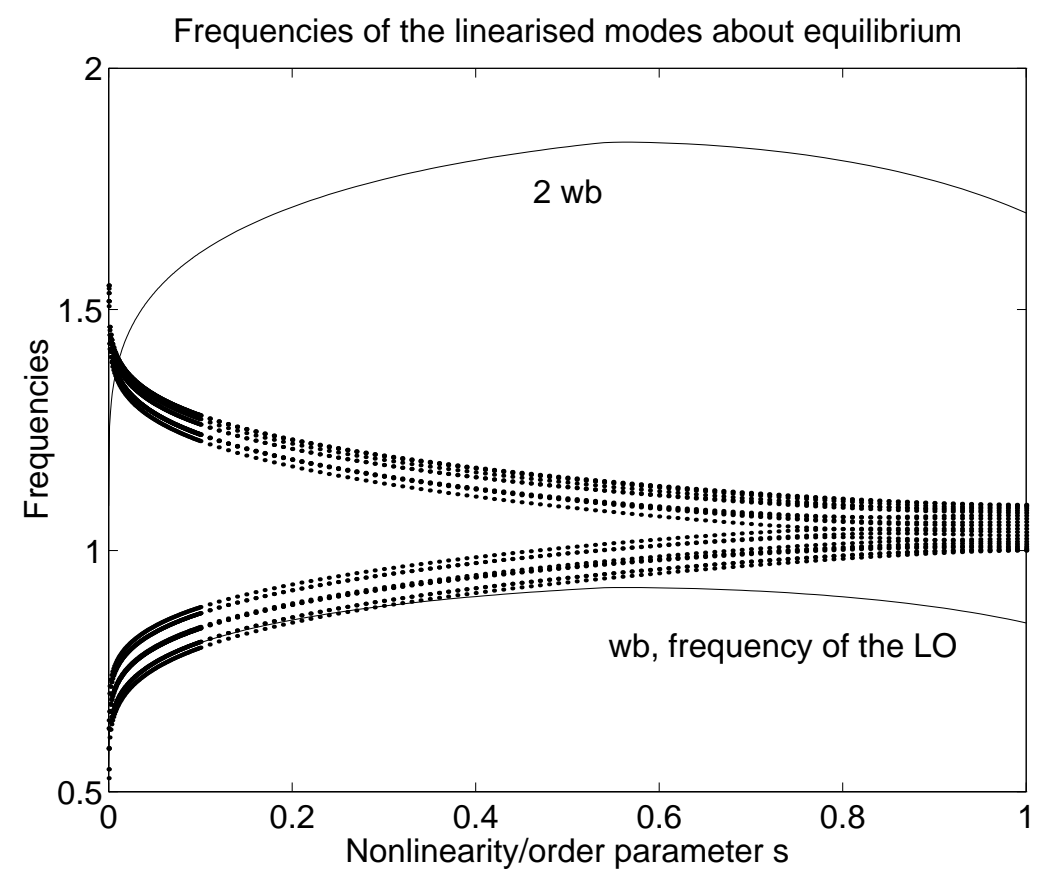

Fig. 11. Frequencies of the LO (also twice its frequency) and all the linearised modes about equilibrium for the $1 / 4$ path.

\subsection{Bifurcations in the linear path}

The bifurcation diagram can be seen in Fig. 12. The bifurcation variable is the projection of the solutions on the centre subspace for bif. 2 . The breather starts at $s=1$ with code +1 on particle 11 (in group C) and follows the path $1(+)$, on which the LOs A and B grow as $s$ decreases. The first eigenvalue crossing the zero line is not visible in Fig. 12, but corresponds to a linear mode on another part of the chain, given a discontinuity like the one described above. The second produces the first important bifurcation, i.e. related to interactions of LOs of non-negligible amplitude. This is bif. 1 , at $s_{1}=0.322$. There the central mode on C, disappears, producing path 2, on which the odd Fourier coefficients are negligible and presumed to be due to numerical error, i.e. the frequency is doubled. Continuation of the solution backwards from the bifurcation leads to path $1(-)$, where the solution is identical to that on path $1(+)$ except for a phase change of $\pi$, leading at $s=1$ to the single breather coded -1 on particle 11. Manipulation of the solutions in path 1, before and near the bifurcation, eliminating the central LO, and continuing it forwards and backwards, makes it possible to find a third branch, path 5, which leads at $s=1$ to a general oscillation of the system.

Putting these together, we obtain an inverse period-doubling bifurcation, shown in the inset to Fig. 12 by using as bifurcation variable a coordinate on the centre subspace for bif. 1 , which distinguishes between the two phases $1( \pm)$. It 


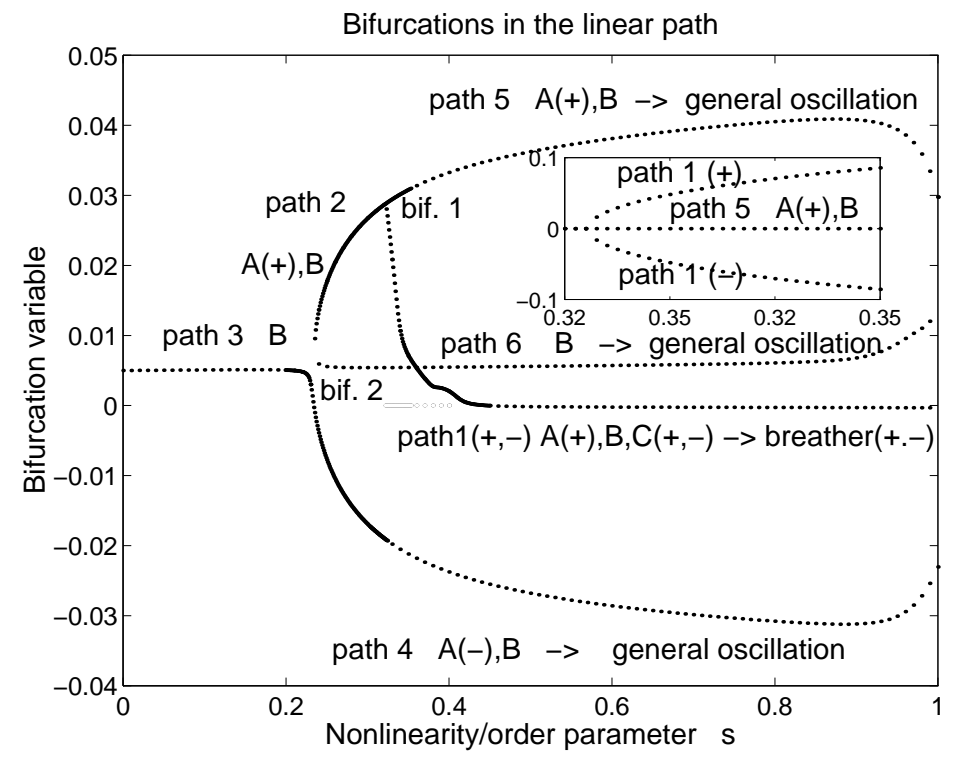

Fig. 12. Bifurcation diagram along the linear path. The bifurcation variable is the component in Fourier space in the direction of the eigenvector with zero eigenvalue for the operator $\partial_{\tilde{z}} \tilde{G}(\tilde{z}, s)$ at bif. 2 (bif. 1 in the inset). It is fully explained in the text.

is not a standard period doubling bifurcation (as described by [25] for generic Hamiltonian systems or [24] for reversible Hamiltonian systems), however, because numerics show that all branches are linearly stable. Our conjecture is that there is a short interval of instability on path 5 which terminates in another period doubling bifurcation, generating an unstable LO which differs from that on path 1 mainly by group $C$ being phase-shifted by $\pi / 2$. This would still be a time-symmetric orbit, but about $t=T / 4$ rather than $t=0$ ( $T$ being the period).

Continuation of path 2 leads to bif. 2 , at $s_{2}=0.234$, where it jumps onto path 3 , where the LO A has disappeared, and at $s=0$, it is the only surviving LO. Using analogous procedures to the above, we also obtain two other nearby branches, giving paths 4 and 6 . On path 4 , the LO A(-) differs from $\mathrm{A}(+)$ by a phase $\pi$, and on path 6 , the LO $\mathrm{A}$ is absent. The limits of paths 4 and 5 leading backwards to general oscillations give, approaching to $s=1$, different oscillations. The approximate symmetry between paths 4 and 5 occurs only near the bifurcation. We identify this bifurcation as an imperfect pitchfork, but again since all the branches shown are linearly stable, there must be some unstable branches too and we conjecture the same diagram as in the previous subsection. 


\subsection{General comment}

In each case, we found only a limited number of eigenvalues crossing zero, which are the eigenvalues of the two or three first linear modes of the particles with frequencies $\omega_{+}$or $\omega_{-}$near rest, and the compound modes, depending on the path followed; their number is of the order of the number of particles. Increasing the number of Fourier components does not change this fact; only the very few first components differ appreciably from zero, and the higher modes are of very small amplitude. Increasing the number of particles, on the other hand, increases proportionally the number of zero crossings. Therefore, we guess that for infinite systems there will be an infinite number of eigenvalues crossing zero.

\section{Conclusion}

Discrete breathers are time-periodic localised oscillations (LO) of weakly coupled networks of anharmonic oscillators. Anderson modes are LOs for spatially random networks with linear dynamics. For a model which interpolates between these two limits, we have found numerically that although Anderson modes typically ${ }^{7}$ evolve to a "general oscillation" with no spatial localisation, on moving parameters in the opposite direction, discrete breathers can be followed almost continuously to Anderson modes, which are often (though not always) localised around the same sites as the breathers. This asymmetry is natural in view of the fact that there are many more time-symmetric periodic solutions of given period at the ordered anharmonic limit $\left(\left(3^{m}-1\right) / 2\right.$ for a system of size $m$ ) than at the disordered harmonic limit $(m)$, but only $m$ of them are single-site breathers. The others are "multi-site breathers" [4].

No paths have been found, however, that completely avoid degeneracies in the linearized continuation operator. We found bifurcations at these points. They are often fold bifurcations, which strictly speaking makes it impossible to continue further. Nevertheless, at every fold point we always found a very nearby path onto which to jump and continue towards the disordered linear limit. For some paths these bifurcations are related to changes in behaviour of groups of particles near rest. This happens, for example, in the path called the $1 / 4$ path. The maximum value of the Fourier coefficients of the particles involved in the bifurcation are about $10^{-2}$ times the largest of the whole system. Even less significant are the eigenvalues of the $k=2$ modes crossing zero almost at

\footnotetext{
7 Not always: in some cases, the computation continued an Anderson mode onto a discrete breather, but this depended on how fast it went through some bifurcation points where there was a "random" choice of branches.
} 
the linear limit where they will disappear. No change of stability is observed in these paths, although it can be found in some others. A detailed study of the main bifurcations has been made, obtaining the different branches and plotting them in numerically determined centre subspaces. We found inverse period doublings and broken pitchforks. Bifurcations such as these, destroying LOs as disorder increases and nonlinearity decreases, should be expected, since there are many more time-symmetric periodic orbits of given action at the ordered anharmonic limit than at the disordered harmonic limit, as already remarked above. A very interesting project for the future is to understand the generic bifurcations for such systems, which are not standard ones because they are unfoldings of bifurcations for decoupled systems.

A technical, but possibly important, point is that we continue LOs at constant action, rather than constant period.

\section{Acknowledgements}

We thank A. C. Scott for useful comments on the manuscript. JLM acknowledges a Marie-Curie TMR fellowship from the EU, no. ERBFMBICT972761.

\section{References}

[1] H Feddersen. Localization of vibrational energy in globular protein. Phys Lett A, 154:391-5, 1991.

[2] A Scott. Davydov's soliton. Phys Rpts, 217:1-67, 1992.

[3] S Flach and CR Willis. Discrete breathers. Phys Rpts, 295:181-264, 1998.

[4] RS MacKay and S Aubry. Proof of existence of breathers for time-reversible or Hamiltonian networks of weakly coupled oscillators. Nonlinearity, 7:1623-1643, 1994.

[5] BV Chirikov. A universal instability of many-dimensional oscillator systems. Phys Reports, 52:263-379, 1979.

[6] S Bolotin and RS MacKay. Isochronous oscillators. in preparation.

[7] S Aubry. Breathers in nonlinear lattices: Existence, linear stability and quantization. Physica D, 103:201-250, 1997.

[8] R Livi, M Spicci, and RS MacKay. Breathers on a diatomic FPU chain. Nonlinearity, 10:1421-34, 1997.

[9] S Aubry. Discrete breathers in anharmonic models with acoustic phonons. Ann IHP Phys Théorique, 4:381-420, 1998. 
[10] PW Anderson. Absence of diffusion in certain random lattices. Phys. Rev., 109:1492-1505, 1958.

[11] TC Spencer. The Schrödinger equation with a random potential. In K Osterwalder and R Stora, editors, Critical Phenomena, Random systems, Gauge theories, Proceedings of the Les Houches Summer School, pages 895943. N. Holland, Amsterdam, 1986.

[12] DJ Thouless. Introduction to disordered systems. In K Osterwalder and R Stora, editors, Critical Phenomena, Random systems, Gauge theories, Proceedings of the Les Houches Summer School, pages 681-. N. Holland, Amsterdam, 1986.

[13] JA Sepulchre and RS MacKay. Discrete breathers in disordered media. Physica D, 113:342-345, 1998.

[14] JL Marin and S Aubry. Breathers in nonlinear lattices: Numerical calculation from the anticontinuous limit. Nonlinearity, 9:1501-1528, 1996.

[15] JL Marin. Intrinsic Localised Modes in Nonlinear Lattices. PhD dissertation, University of Zaragoza, Department of Condensed Matter, June 1997.

[16] JC Eilbeck, PS Lomdahl, and AC Scott. The discrete self-trapping equation. Physica D, 16:318-338, 1985.

[17] G Kopidakis and S Aubry. Intraband discrete breathers in disordered nonlinear systems i: Delocalization. Preprint, Saclay, 20 Nov 98.

[18] C Albanese and J Fröhlich. Perturbation theory for periodic orbits in a class of infinite dimensional hamiltonian systems. Commun Math Phys, 138:193-205, 1991.

[19] RS MacKay and JA Sepulchre. Stability of discrete breathers. Physica D, 119:148-162, 1998.

[20] JL Marin and S Aubry. Breathers in nonlinear lattices: Numerical methods based on the anti-integrability concept. In L Vázquez, L Streit, and VM PérezGarcía, editors, Nonlinear Klein-Gordon and Schrödinger Systems: Theory and Applications, pages 317-323. World Scientific, Singapore and Philadelphia, 1995 .

[21] J Frölich, T Spencer, and CE Wayne. Localization in disordered, nonlinear dynamical systems. J. Statist. Phys., 42:247-274, 1986.

[22] J Carr and JC Eilbeck. Stability of stationary solutions of the discrete selftrapping equation. Phys Lett A, 109:201-204, 1985.

[23] JL Marin and S Aubry. Finite size effects on instabilities of discrete breathers. Physica D, 119:163-174, 1998.

[24] R Rimmer. Generic bifurcation for involutory area preserving maps. Mem Am Math Soc, 41:1-165, 1983.

[25] KR Meyer. Generic bifurcation of periodic points. Trans Am Math Soc, 149:95107, 1970. 